ISSN 1855-3966 (printed edn.), ISSN 1855-3974 (electronic edn.)

ARS MATHEMATICA CONTEMPORANEA 17 (2019) 525-533

https://doi.org/10.26493/1855-3974.1610.03d

(Also available at http://amc-journal.eu)

\title{
On the Hamilton-Waterloo problem: the case of two cycles sizes of different parity*
}

\author{
Melissa S. Keranen \\ Michigan Technological University, Department of Mathematical Sciences, \\ Houghton, MI 49931, USA \\ Adrián Pastine \\ Instituto de Matemática Aplicada San Luis (IMASL), Universidad Nacional de San Luis, \\ CONICET, Ejército de los Andes 950 (D5700HHW), San Luis, Argentina
}

Received 19 February 2018, accepted 3 October 2019, published online 25 November 2019

\begin{abstract}
The Hamilton-Waterloo problem asks for a decomposition of the complete graph of order $v$ into $r$ copies of a 2 -factor $F_{1}$ and $s$ copies of a 2 -factor $F_{2}$ such that $r+s=\left\lfloor\frac{v-1}{2}\right\rfloor$. If $F_{1}$ consists of $m$-cycles and $F_{2}$ consists of $n$ cycles, we say that a solution to $(m, n)$ $\operatorname{HWP}(v ; r, s)$ exists. The goal is to find a decomposition for every possible pair $(r, s)$. In this paper, we show that for odd $x$ and $y$, there is a solution to $\left(2^{k} x, y\right)-\operatorname{HWP}(v m ; r, s)$ if $\operatorname{gcd}(x, y) \geq 3, m \geq 3$, and both $x$ and $y$ divide $v$, except possibly when $1 \in\{r, s\}$.

Keywords: 2-factorizations, Hamilton-Waterloo problem, Oberwolfach problem, cycle decomposition, resolvable decompositions.
\end{abstract}

Math. Subj. Class.: 05C51, 05C70

\section{Introduction}

The Oberwolfach problem asks for a decomposition of the complete graph $K_{v}$ into $\frac{v-1}{2}$ copies of a 2 -factor $F$. To achieve this decomposition, $v$ needs to be odd, because the vertices must have even degree. The problem with $v$ even asks for a decomposition of $K_{v}$ into $\frac{v-2}{2}$ copies of a 2 -factor $F$, and one copy of a 1 -factor. The uniform Oberwolfach problem (all cycles of the 2 -factor have the same size) has been completely solved by

*The authors would like to thank Stefaan De Winter and Martín De Borbón for wonderful discussions about rings of polynomials that led to the original constructions used in previous versions of the paper, and the anonymous referees for helpful comments which greatly improved the readability of the final version.

This work was partially supported by the Universidad Nacional de San Luis, grant PROICO 03-0918.

E-mail addresses: msjukuri@mtu.edu (Melissa S. Keranen), adrian.pastine.tag@gmail.com (Adrián Pastine) 
Alspach and Haggkvist [1] and Alspach, Schellenberg, Stinson and Wagner [2]. The nonuniform Oberwolfach problem has been studied as well, and a survey of results up to 2006 can be found in [8]. Furthermore, one can refer to [6, 7, 9, 23, 24] for more recent results.

In [19] Liu first worked on the generalization of the Oberwolfach problem to equipartite graphs. He was seeking to decompose the complete equipartite graph $K_{(m: n)}$ with $n$ partite sets of size $m$ each into $\frac{(n-1) m}{2}$ copies of a 2 -factor $F$. For such a decomposition to exist $(n-1) m$ has to be even. In [14] Hoffman and Holliday worked on the equipartite generalization of the Oberwolfach problem when $(n-1) m$ is odd, decomposing into $\frac{(n-1) m-1}{2}$ copies of a 2 -factor $F$, and one copy of a 1-factor. The uniform Oberwolfach problem over equipartite graphs has since been completely solved by Liu [20] and Hoffman and Holliday [14]. For the non-uniform case, Bryant, Danziger and Pettersson [7] completely solved the case when the 2-factor is bipartite. In particular, Liu showed the following.

Theorem 1.1 ([20]). For $m \geq 3$ and $u \geq 2, K_{(h: u)}$ has a resolvable $C_{m}$-factorization if and only if hu is divisible by $m, h(u-1)$ is even, $m$ is even if $u=2$, and $(h, u, m) \notin$ $\{(2,3,3),(6,3,3),(2,6,3),(6,2,6)\}$.

The Hamilton-Waterloo problem is a variation of the Oberwolfach problem, in which we consider two 2-factors, $F_{1}$ and $F_{2}$. It asks for a factorization of $K_{v}$ when $v$ is odd or $K_{v}-I$ ( $I$ is a 1-factor) when $v$ is even into $r$ copies of $F_{1}$ and $s$ copies of $F_{2}$ such that $r+s=\left\lfloor\frac{v-1}{2}\right\rfloor$, where $F_{1}$ and $F_{2}$ are two 2-regular graphs on $v$ vertices. Most of the results for the Hamilton-Waterloo problem are uniform, meaning $F_{1}$ consists of cycles of size $m$ ( $C_{m}$-factors), and $F_{2}$ consists of cycles of size $n\left(C_{n}\right.$-factors). If there is a decomposition of $K_{v}$ into $r C_{m}$-factors and $s C_{n}$-factors we say that a solution to $(m, n)-\operatorname{HWP}(v ; r, s)$ exists. The case where both $m$ and $n$ are odd positive integers and $v$ is odd is almost completely solved by [11,12]; and if $m$ and $n$ are both even, then the problem again is almost completely solved (see [5, 6]). However, if $m$ and $n$ are of differing parities, then we only have partial results. Most of the work has been done in the case where one of the cycle sizes is constant. The case of $(m, n)=(3,4)$ is solved in $[4,13,21,25]$. Other cases which have been studied include $(m, n)=(3, v)$ [18], $(m, n)=(3,3 x)$ [3], and $(m, n)=(4, n)[16,21]$.

In this paper, we consider the case of $m$ and $n$ being of different parity. This case has gained attention recently, where it has been shown that the necessary conditions are sufficient for a solution to $(m, n)-\operatorname{HWP}(v ; r, s)$ to exist whenever $m \mid n, v>6 n>36 m$, and $s \geq 3$ [10]. We provide a complementary result to this in our main theorem, which covers cases in which $m \nmid n$ and solves a major portion of the problem.

Theorem 1.2. Let $x, y, v, k$ and $m$ be positive integers such that:

(i) $v, m \geq 3$,

(ii) $x, y$ are odd,

(iii) $\operatorname{gcd}(x, y) \geq 3$,

(iv) $x$ and $y$ divide $v$,

(v) $4^{k}$ divides $v$.

Then there exists a solution to $\left(2^{k} x, y\right)-\operatorname{HWP}(v m ; r, s)$ for every pair $r, s$ with $r+s=$ $\lfloor(v m-1) / 2\rfloor, r, s \neq 1$. 


\section{Preliminaries}

The complete cyclic multipartite graph $C_{(x: n)}$ is the graph with $n$ partite sets of size $x$, where two vertices $(g, i)$ and $(h, j)$ are neighbors if and only if $i-j= \pm 1(\bmod n)$, with subtraction being done modulo $n$. The directed complete cyclic multipartite graph $\vec{C}_{(x: n)}$ is the graph with $n$ parts of size $x$, with arcs of the form $((g, i),(h, i+1))$ for every $0 \leq g, h \leq x-1,0 \leq i \leq n-1$.

One of the main tools in [17] is a Lemma that combines decompositions of $C_{(x: k)}$ to obtain decompositions of $K_{(v: m)}$. We present a version of the Lemma for uniform decompositions, as those are the focus of this manuscript.

Lemma 2.1 ([17]). Let $m, x, y$, and $v$ be positive integers. Let $s_{1}, \ldots, s_{\frac{m-1}{2}}$ be nonnegative integers. Suppose the following conditions are satisfied:

- There exists a decomposition of $K_{m}$ into $C_{n}$-factors.

- For every $1 \leq t \leq \frac{m-1}{2}$ there exists a decomposition of $C_{(v: n)}$ into $s_{t} C_{x n}$-factors and $r_{t} C_{y n}$-factors.

Let

$$
s=\sum_{t=1}^{\frac{(m-1)}{2}} s_{t} \quad \text { and } \quad r=\sum_{t=1}^{\frac{(m-1)}{2}} r_{t} .
$$

Then there exists a decomposition of $K_{(v: m)}$ into $s C_{x n}$-factors and $r C_{y n}$-factors.

In order to decompose $\vec{C}_{(x: n)}, x$ and $n$ odd, into $C_{n}$-factors and $C_{x n}$-factors, the authors of [17] labeled the vertices by $\mathbb{Z}_{x} \times\{0, \ldots, n-1\}$. They build a 2 -factor $F$ by providing $n$ permutations of $G$. The $i$ th permutation is used to connect vertices in column $i-1$ to vertices in column $i$, in particular the $n$-th permutation is used to connect vertices in column $n-1$ to vertices in column 0 . It must be said that these permutations were used implicitly in [17], as no permutation language was used for this part of the construction.

Notice that in general, if the columns are labeled by an abelian group $G, f$ is the $i$ th permutation and $g \in G$, in the 2 -factor $F$, vertex $(g, i-1)$ is connected to vertex $(f(g), i)$. Let $\mathcal{F}$ be the composition of all $n$ permutations of the 2-factor $F$, such that $(\mathcal{F}(g), 0)$ is the vertex at which we finish if we start at vertex $(g, 0)$ and move through $F$ until we reach column 0 again. In the constructions in [17], $G$ is abelian, and $g-\mathcal{F}(g)$ depends only on $\mathcal{F}$ and not on $g$. If this is the case, the length of the cycles of $F$ is $n$ times the order of the element $g-\mathcal{F}(g)$.

Lemma 2.2. Assume $F$ is a 2-factor built with the permutation $\mathcal{F}$, and $g-\mathcal{F}(g)$ depends only on $\mathcal{F}$. If $q$ is the order of $g-\mathcal{F}(g)$, then $F$ is a $\vec{C}_{q n}$-factor of $\vec{C}_{\left(x y 4^{k}: n\right)}$.

As we will need to use the permutations of $\mathbb{Z}_{x}$, we will introduce them. For $\alpha \in \mathbb{Z}_{x}$, let $f_{\alpha}$ be the permutation that adds $\alpha$ to every element of $\mathbb{Z}_{x}$, i.e. $f_{\alpha}(g)=g+\alpha$. Let $H(\alpha, \beta)$ be the 2-factor made with the following permutations:

- $f_{\alpha}$ from column $i-1$ to column $i$ if $1 \leq i \leq n-3 / 2$;

- $f_{-\alpha}$ from column $i-1$ to column $i$ if $n-3 / 2+1 \leq i \leq n-3$;

- $f_{\alpha}$ from column $n-3$ to column $n-2$; 
- $f_{-2 \alpha}$ from column $n-2$ to column $n-1$ (this is a permutation because $x$ is odd);

- $f_{\beta}$ from column $n-1$ to column 0 .

In [17] the first $n-3$ permutations were different, but the end result was the same. Notice that $\mathcal{F}(g)=g-\alpha+\beta$. For every $r \in\{0,1,2, \ldots, x-3, x-2, x\}$, the authors of [17] gave permutations $\phi$ of $\mathbb{Z}_{x}$ that satisfied:

(a) $\phi(\alpha)=\alpha$ for $r$ elements of $\mathbb{Z}_{x}$;

(b) $\operatorname{gcd}(\alpha-\phi(\alpha), x)=1$ for the remaining $x-r$ elements of $\mathbb{Z}_{x}$.

Then, the decomposition of $\vec{C}_{(x: n)}$ was given by the 2-factors $H(\alpha, \phi(\alpha)), \alpha \in \mathbb{Z}_{x}$.

In order for such a decomposition to work, for every $\alpha, \beta \in \mathbb{Z}_{x}$ the permutations $f_{\alpha}, f_{\beta}$ needed to satisfy $f_{\alpha}=f_{\beta}$ if and only if $\alpha=\beta$, as otherwise some arcs would be repeated in the factor $H(\alpha, \phi(\alpha))$ and the factor $H(\beta, \phi(\beta))$.

Then, in [17], decompositions of $\vec{C}_{(x: n)}, \vec{C}_{(y: n)}$, and $\vec{C}_{(4: n)}$ were combined using a graph product and permutations of $\mathbb{Z}_{x} \times \mathbb{Z}_{y} \times \mathbb{Z}_{4}$ to decompose $\vec{C}_{(4 x y: n)}$. Instead of doing so, we will use group products to label the vertices of $\vec{C}_{\left(4^{k} x y: n\right)}$, although we will make use of permutations of the group product.

In Section 3, we give permutations of $\mathbb{Z}_{2^{k}} \times \mathbb{Z}_{2^{k}}$, and show that they satisfy the necessary conditions to be used for decompositions. In Section 4, we use multivariate bijections to give decompositions of $\vec{C}_{\left(4^{k} x y: n\right)}$ into $\vec{C}_{2^{k} x k}$-factors and $\vec{C}_{y k}$-factors. Finally, in Section 5 , we use these decompositions to prove our main results.

\section{The permutation $f_{\alpha}(a, b)$ of $\mathbb{Z}_{2^{k}} \times \mathbb{Z}_{2^{k}}$}

Consider the group $G=\mathbb{Z}_{2^{k}} \times \mathbb{Z}_{2^{k}}$, an element $\alpha=\left(\alpha_{1}, \alpha_{2}\right)$ and the function $f_{\alpha}(a, b)=$ $\left(-b+\alpha_{1}, a-b+\alpha_{2}\right)$.

Lemma 3.1. $f_{\alpha}$ is a permutation of $G$.

Proof. As $|G|$ is finite, it is enough to prove that $f_{\alpha}$ is an injective function.

Assume $f_{\alpha}(a, b)=f_{\alpha}(c, d)$. Then

$$
\left(-b+\alpha_{1}, a-b+\alpha_{2}\right)=\left(-d+\alpha_{1}, c-d+\alpha_{2}\right) .
$$

The equality $-b+\alpha_{1}=-d+\alpha_{1}$ implies $b=d$. Using $b=d$, the equality $a-b+\alpha_{2}=$ $c-d+\alpha_{2}$ implies $a=c$. Therefore, $f_{\alpha}$ is a permutation of $G$.

Lemma 3.2. $f_{\beta}\left(f_{\alpha}^{2}(a, b)\right)=(a, b)-\alpha+\beta$.

Proof. We will prove this lemma by computing $f_{\beta}\left(f_{\alpha}^{2}(a, b)\right)$.

$$
\begin{aligned}
f_{\alpha}(a, b) & =\left(-b+\alpha_{1}, a-b+\alpha_{2}\right) \\
f_{\alpha}^{2}(a, b) & =f\left(-b+\alpha_{1}, a-b+\alpha_{2}\right) \\
& =\left(-a+b-\alpha_{2}+\alpha_{1},-b+\alpha_{1}-a+b-\alpha_{2}+\alpha_{2}\right) \\
& =\left(-a+b-\alpha_{2}+\alpha_{1}, \alpha_{1}-a\right)
\end{aligned}
$$




$$
\begin{aligned}
f_{\beta}\left(f_{\alpha}^{2}(a, b)\right) & =f_{\beta}\left(-a+b-\alpha_{2}+\alpha_{1}, \alpha_{1}-a\right) \\
& =\left(a-\alpha_{1}+\beta_{1},-a+b-\alpha_{2}+\alpha_{1}-\alpha_{1}+a+\beta_{2}\right) \\
& =\left(a-\alpha_{1}+\beta_{1}, b-\alpha_{2}+\beta_{2}\right) \\
& =(a, b)-\alpha+\beta .
\end{aligned}
$$

Letting $\beta=\alpha$ in Lemma 3.2 yields $f_{\alpha}^{3}(a, b)=(a, b)$.

Corollary 3.3. $f_{\alpha}^{3}(a, b)=(a, b)$.

As it was mentioned in Section 2, we need to show that if $\alpha \neq \beta$, then $f_{\alpha}(a, b) \neq$ $f_{\beta}(a, b)$ for every $(a, b) \in \mathbb{Z}_{2^{k}} \times \mathbb{Z}_{2^{k}}$; so that each arc is used exactly once. The statement of the following lemma is an equivalent claim.

Lemma 3.4. $f_{\alpha}(a, b)=f_{\beta}(a, b)$ for some $(a, b) \in \mathbb{Z}_{2^{k}} \times \mathbb{Z}_{2^{k}}$ if and only if $\alpha=\beta$.

Proof. Assume $f_{\alpha}(a, b)=f_{\beta}(a, b)$. Then

$$
\left(-b+\alpha_{1}, a-b+\alpha_{2}\right)=\left(-b+\beta_{1}, a-b+\beta_{2}\right) .
$$

Hence, $\alpha_{1}=\beta_{1}$ and $\alpha_{2}=\beta_{2}$. Therefore $\alpha=\beta$.

\section{Decomposing $\vec{C}_{\left(4^{k} x y: n\right)}$ into $\vec{C}_{y n}$-factors and $\vec{C}_{x 2^{k} n}$-factors}

Let $G=\mathbb{Z}_{2^{k}} \times \mathbb{Z}_{2^{k}}$ and label each column of $\vec{C}_{\left(4^{k} x y: n\right)}$ with the elements of the group $G \times \mathbb{Z}_{x} \times \mathbb{Z}_{y}$.

Let $R=G \times \mathbb{Z}_{x} \times \mathbb{Z}_{y}$. For every $\lambda \in R$, let $\lambda=(\alpha, \beta, \gamma)$, with $\alpha \in G, \beta \in \mathbb{Z}_{x}$ and $\gamma \in \mathbb{Z}_{y}$. For $\alpha \in G$, let $f_{\alpha}$ be defined as in Section 3. For $\beta \in \mathbb{Z}_{x}$ let $f_{\beta}$ be the permutation of $\mathbb{Z}_{x}$ defined by $f_{\beta}(a)=a+\beta$. Similarly, for $\gamma \in \mathbb{Z}_{y}$ let $f_{\gamma}$ be the permutation of $\mathbb{Z}_{y}$ defined by $f_{\gamma}(a)=a+\gamma$. Finally, for $\lambda=(\alpha, \beta, \gamma) \in R$ let $f_{\lambda}$ be the permutation of $R$ defined by $f_{\lambda}(a, b, c)=\left(f_{\alpha}(a), f_{\beta}(b), f_{\gamma}(c)\right)$.

Let $\varphi$ be a permutation of $R$, and for each $\lambda \in G$ let $H_{4^{k} x y}(\lambda, \varphi(\lambda))$ be the 2-factor formed with the following permutations:

1. $f_{\lambda}$ from column $i$ to $i+1$ if $1 \leq i \leq n-3 / 2$;

2. $f_{\lambda}^{-1}$ from column $i$ to $i+1$ if $n-3 / 2+1 \leq i \leq n-3$;

3. $f_{\lambda}$ from column $n-2$ to column $n-1$;

4. $f(\alpha,-2 \beta,-2 \gamma)$ from column $n-1$ to column $n$;

5. $f_{\varphi(\alpha)}$ from column $n$ to column 1 .

Notice that if you start in column 1 at vertex $(a, b, c)$ the first time you reach column 1 again you reach vertex

$$
(a, b, c)-(\alpha, \beta, \gamma)+\varphi(\alpha, \beta, \gamma)=(a, b, c)-\lambda+\varphi(\lambda)
$$

Hence, we can apply Lemma 2.2 to obtain the length of the cycles in the 2 -factor.

Let $\lambda \in R$. If $\lambda-\varphi(\lambda)=(a, b, 0)$ with $a \in \pm\{(1,0),(0,1),(1,1)\}$ and $\operatorname{gcd}(b, x)=1$, then by Lemma 2.2 the 2 -factor $H_{4^{k}}(\lambda, \varphi(\lambda))$ is a $\vec{C}_{2^{k} x n}$-factor. If $\lambda-\varphi(\lambda)=(0,0, c)$ with $\operatorname{gcd}(c, y)=1$, Lemma 2.2 implies that $H_{4^{k}}(\lambda, \varphi(\lambda))$ is a $\vec{C}_{y n}$-factor.

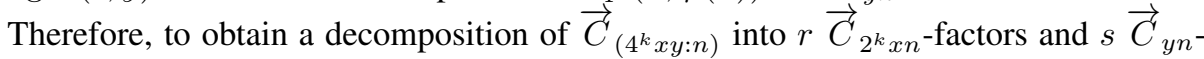
factors, we need a permutation $\varphi$ satisfying 
(A) $\lambda-\varphi(\lambda)=(a, b, 0)$ with $a \in \pm\{(1,0),(0,1),(1,1)\}$ and $\operatorname{gcd}(b, x)=1$ for $r$ elements $\lambda \in R$;

(B) $\lambda-\varphi(\lambda)=(0,0, c)$ with $\operatorname{gcd}(c, y)=1$ for $s=4^{k} x y-r$ elements $\lambda \in R$.

In order to obtain the permutation $\varphi$, consider the subgroup $2 G$ of $G$ of index 4 , and let

$$
K=\{(0,0),(1,0),(0,1),(1,1)\} .
$$

Notice that $K$ is a set of representatives of the cosets of $2 G$ in $G$. Let $\epsilon \in 2 G$, and let $\phi$ be a permutation of $G$. If $g, \phi(g) \in \epsilon+K$, then either $g=\phi(g)$ or $|g-\phi(g)|=2^{k}$ because $g-\phi(g) \in \pm K$. Hence, we can obtain $\varphi$ by providing $4^{k-1}$ permutations $\rho_{\epsilon}$ of $K \times \mathbb{Z}_{x} \times \mathbb{Z}_{y}$ satisfying

$\left(\mathrm{A}^{\prime}\right) \lambda-\rho_{\epsilon}(\lambda)=(a, b, 0)$ with $a \in \pm\{(1,0),(0,1),(1,1)\}$ and $\operatorname{gcd}(b, x)=1$ for $r_{\epsilon}$ elements $\lambda \in K \times \mathbb{Z}_{x} \times \mathbb{Z}_{y}$

(B') $\lambda-\rho_{\epsilon}(\lambda)=(0,0, c)$ with $\operatorname{gcd}(c, y)=1$ for $s_{\epsilon}=4 x y-r_{\epsilon}$ elements $\lambda \in K \times \mathbb{Z}_{x} \times \mathbb{Z}_{y}$;

having $r=\sum_{\epsilon \in 2 G} r_{\epsilon}$, and having $\varphi$ act in each $(\epsilon+K) \times \mathbb{Z}_{x} \times \mathbb{Z}_{y}$ as $\rho_{\epsilon}$, i.e. if $g=$ $(\epsilon, c, d)+(\mu, 0,0)$, with $\mu \in K, \varphi(g)=(\epsilon, c, d)+\rho_{\epsilon}(\mu, c, d)$. Notice that if $a \in K$, $a \in \pm\{(1,0),(0,1),(1,1)\}$ if and only if $a \neq(0,0)$.

In [17], for every $r \in\{0,2,3, \ldots, 4 x y-3,4 x y-2,4 x y\}$, permutations $\phi$ of $\mathbb{Z}_{4} \times$ $\mathbb{Z}_{x} \times \mathbb{Z}_{y}$ were given satisfying:

$\left(\mathrm{A}^{\prime \prime}\right) \lambda-\phi(\lambda)=(a, b, 0)$, with $a \neq 0$ and $\operatorname{gcd}(b, x)=1$ for $r$ elements $\lambda \in \mathbb{Z}_{4} \times \mathbb{Z}_{x} \times \mathbb{Z}_{y}$; $\left(\mathrm{B}^{\prime \prime}\right) \lambda-\phi(\lambda)=(0,0, c)$, with $\operatorname{gcd}(c, y)=1$ for the remaining $4 x y-r$ elements $\lambda \in$ $\mathbb{Z}_{4} \times \mathbb{Z}_{x} \times \mathbb{Z}_{y}$

Let $\pi: K \rightarrow \mathbb{Z}_{4}$ be a bijection such that $\pi(0,0)=0$, and let $\psi: K \times \mathbb{Z}_{x} \times \mathbb{Z}_{y} \rightarrow$ $\mathbb{Z}_{4} \times \mathbb{Z}_{x} \times \mathbb{Z}_{y}$ be the bijection that fixes the coordinates of $\mathbb{Z}_{x}$ and $\mathbb{Z}_{y}$, and that behaves like $\pi$ in the coordinate of $K$. Then if $\phi_{\epsilon}$ is a permutation of $\mathbb{Z}_{4} \times \mathbb{Z}_{x} \times \mathbb{Z}_{y}$, satisfying Conditions $\left(\mathrm{A}^{\prime \prime}\right)$ and $\left(\mathrm{B}^{\prime \prime}\right)$ with $r_{\epsilon}$ and $s_{\epsilon}, \rho_{\epsilon}=\psi^{-1} \phi_{\epsilon} \psi$ is a permutation of $K \times \mathbb{Z}_{x} \times \mathbb{Z}_{y}$ satisfying Conditions $\left(\mathrm{A}^{\prime}\right)$ and $\left(\mathrm{B}^{\prime}\right)$ with $r_{\epsilon}$ and $s_{\epsilon}$.

If we wanted either $x=1$ or $y=1$, we would need to change Conditions (A) and (B), but it is easy to see that the necessary permutations to decompose $\vec{C}_{\left(4^{k} x y: n\right)}$ exist.

Therefore we have the following.

Lemma 4.1. Let $r \notin\left\{1,4^{k} x y-1\right\}$, then there is a decomposition of $\vec{C}_{\left(4^{k} x y: n\right)}$ into $r$ $\vec{C}_{2^{k} x n}$-factors and $s=4^{k} x y-r C_{y n}$-factors.

\section{Main results}

The complete solution to the uniform case of the Oberwolfach problem will be vital to the proof of our main result.

Theorem 5.1 ([1, 2, 15, 22]). $K_{v}$ can be decomposed into $C_{m}$-factors (and a 1-factor if $v$ is even) if and only if $v \equiv 0(\bmod m),(v, m) \neq(6,3)$ and $(v, m) \neq(12,3)$.

We now apply the results from Section 4 to produce the following important result for the uniform equipartite version of the Hamilton-Waterloo problem where the two factor types consist of cycle sizes of distinct parities. 
Theorem 5.2. Let $x, y, z, v, m, k$ be positive integers $v, m, k \geq 3$ satisfying the following:

(i) $v, m \geq 3$,

(ii) $k \geq 2$,

(iii) $x, y, z$ odd,

(iv) $z \geq 3$,

(v) $\operatorname{gcd}(x, y)=1$,

(vi) $v m \equiv 0\left(\bmod 4^{k} x y z\right), v \equiv 0\left(\bmod 4^{k} x y\right)$,

(vii) $\frac{v(m-1)}{4^{k} x y}$ is even,

(viii) $\left(\frac{v}{4^{k} x y}, m, z\right) \notin\{(2,3,3),(6,3,3),(2,6,3),(6,2,6)\}$,

then there is a decomposition of $K_{(v: m)}$ into $r C_{2^{k} x z}$-factors and $s C_{y z}$-factors, for any $s, r \neq 1$.

Proof. Let $v_{1}=v / 4^{k} x y$. Consider $K_{\left(v_{1}: m\right)}$. Item (vi) ensures that $z$ divides $v_{1} m$; and items (vii), (i), and (viii) give us $v_{1}(m-1)$ is even, $m \neq 2$, and

$$
\left(\frac{v}{4^{k} x y}, m, z\right) \notin\{(2,3,3),(6,3,3),(2,6,3),(6,2,6)\} .
$$

Thus by Theorem 1.1 there is a decomposition of $K_{\left(v_{1}: m\right)}$ into $C_{z}$-factors.

Replace each vertex in $a \in K_{\left(v_{1}: m\right)}$ by $4^{k} x y$ vertices $(a, b)$, with $0 \leq b \leq 4^{k} x y-1$, having an edge between $\left(a_{1}, b_{1}\right)$ and $\left(a_{2}, b_{2}\right)$ if and only if there was an edge between $a_{1}$ and $a_{2}$. This yields $K_{(v: m)}$. Even more, each $C_{z}$-factor becomes a copy of $\frac{v_{1} m}{z} C_{\left(4^{k} x y: z\right)}$. By Lemma 4.1, we have that each $\frac{v_{1} m}{z} C_{\left(4^{k} x y: z\right)}$ can be decomposed into $r_{p} C_{2^{k} x z^{-} \text {-factors }}$ and $s_{p} C_{y z}$-factors as long as $r_{p}, s_{p} \neq 1$. Choosing $s_{p}$ such that $\sum_{p} s_{p}=s$ and $s_{p}, r_{p} \neq 1$, provides a decomposition of $K_{(v: m)}$ into $r C_{2^{k} x z}$-factors and $s C_{y z}$-factors by Lemma 2.1.

The next lemma, given in [17] shows how to find solutions to the Hamilton-Waterloo problems by combining solutions for the problem on complete graphs and solutions for the problem on equipartite graphs.

Lemma 5.3 ([17]). Let $m$ and $v$ be positive integers. Let $F_{1}$ and $F_{2}$ be two 2-factors on $v m$ vertices. Suppose the following conditions are satisfied:

- There exists a decomposition of $K_{(v: m)}$ into $s_{\alpha}$ copies of $F_{1}$ and $r_{\alpha}$ copies of $F_{2}$.

- There exists a decomposition of $m K_{v}$ into $s_{\beta}$ copies of $F_{1}$ and $r_{\beta}$ copies of $F_{2}$.

Then there exists a decomposition of $K_{v m}$ into $s=s_{\alpha}+s_{\beta}$ copies of $F_{1}$ and $r=r_{\alpha}+r_{\beta}$ copies of $F_{2}$.

We are now in a position to provide a proof of the main theorem.

Theorem 5.4. Let $x, y, v, k$ and $m$ be positive integers such that:

(i) $v, m \geq 3$,

(ii) $x, y$ are odd, 
(iii) $\operatorname{gcd}(x, y) \geq 3$,

(iv) $x$ and $y$ divide $v$,

(v) $4^{k}$ divides $v$.

Then there exists a solution to $\left(2^{k} x, y\right)-\operatorname{HWP}(v m ; r, s)$ for every pair $r, s$ with $r+s=$ $\lfloor(v m-1) / 2\rfloor, r, s \neq 1$.

Proof. Let $r$ and $s$ be positive integers with $r+s=\lfloor(v m-1) / 2\rfloor$ and $r, s \neq 1$. Write $r=r_{\alpha}+r_{\beta}$ and $s=s_{\alpha}+s_{\beta}$, where $r_{\alpha}, r_{\beta}, s_{\alpha}, s_{\beta}$ are positive integers that satisfy $r_{\alpha}, s_{\alpha} \neq 1, r_{\alpha}+s_{\alpha}=v(m-1) / 2, r_{\beta}+s_{\beta}=\lfloor(v-1) / 2\rfloor$, and $r_{\beta}, s_{\beta} \in\{0,\lfloor(v-1) / 2\rfloor\}$.

Start by decomposing $K_{v m}$ into $K_{(v: m)} \oplus m K_{v}$. Let $z=\operatorname{gcd}(x, y), x_{1}=x / z$, $y_{1}=y / z$. By Theorem 5.2 there is a decomposition of $K_{(v: m)}$ into $r_{\alpha} C_{2^{k} x_{1} z}$-factors and $s_{\alpha} C_{y_{1} z}$-factors. This is a decomposition of $K_{(v: m)}$ into $r_{\alpha} C_{2^{k}}$-factors and $s_{\alpha} C_{y}$-factors. By Theorem 5.1 there is a decomposition of $m K_{v}$ into $r_{\beta} C_{2^{k}}$-factors and $s_{\beta} C_{y}$-factors. Lemma 5.3 shows that all of this together yields a decomposition of $K_{v m}$ into $r C_{x}$-factors and $s C_{y}$-factors.

\section{References}

[1] B. Alspach and R. Häggkvist, Some observations on the Oberwolfach problem, J. Graph Theory 9 (1985), 177-187, doi:10.1002/jgt.3190090114.

[2] B. Alspach, P. J. Schellenberg, D. R. Stinson and D. Wagner, The Oberwolfach problem and factors of uniform odd length cycles, J. Comb. Theory Ser. A 52 (1989), 20-43, doi:10.1016/ 0097-3165(89)90059-9.

[3] J. Asplund, D. Kamin, M. Keranen, A. Pastine and S. Özkan, On the Hamilton-Waterloo problem with triangle factors and $C_{3 x}$-factors, Australas. J. Combin. 64 (2016), 458-474, https://ajc.maths.uq.edu.au/pdf/64/ajc_v64_p458.pdf.

[4] S. Bonvicini and M. Buratti, Octahedral, dicyclic and special linear solutions of some Hamilton-Waterloo problems, Ars Math. Contemp. 14 (2018), 1-14, doi:10.26493/1855-3974. 1088.414 .

[5] D. Bryant and P. Danziger, On bipartite 2-factorizations of $K_{n}-I$ and the Oberwolfach problem, J. Graph Theory 68 (2011), 22-37, doi:10.1002/jgt.20538.

[6] D. Bryant, P. Danziger and M. Dean, On the Hamilton-Waterloo problem for bipartite 2-factors, J. Combin. Des. 21 (2013), 60-80, doi:10.1002/jcd.21312.

[7] D. Bryant, P. Danziger and W. Pettersson, Bipartite 2-factorizations of complete multipartite graphs, J. Graph Theory 78 (2015), 287-294, doi:10.1002/jgt.21806.

[8] D. Bryant and C. A. Rodger, Cycle decompositions, in: C. J. Colbourn and J. H. Dinitz (eds.), Handbook of Combinatorial Designs, CRC Press, Boca Raton, Discrete Mathematics and Its Applications, pp. 373-382, 2nd edition, 2007, doi:10.1201/9781420010541.

[9] D. Bryant and V. Scharaschkin, Complete solutions to the Oberwolfach problem for an infinite set of orders, J. Comb. Theory Ser. B 99 (2009), 904-918, doi:10.1016/j.jctb.2009.03.003.

[10] A. C. Burgess, P. Danziger and T. Traetta, private communication.

[11] A. C. Burgess, P. Danziger and T. Traetta, On the Hamilton-Waterloo problem with odd orders, J. Combin. Des. 25 (2017), 258-287, doi:10.1002/jcd.21552.

[12] A. C. Burgess, P. Danziger and T. Traetta, On the Hamilton-Waterloo problem with odd cycle lengths, J. Combin. Des. 26 (2018), 51-83, doi:10.1002/jcd.21586. 
[13] P. Danziger, G. Quattrocchi and B. Stevens, The Hamilton-Waterloo problem for cycle sizes 3 and 4, J. Combin. Des. 17 (2009), 342-352, doi:10.1002/jcd.20219.

[14] D. G. Hoffman and S. H. Holliday, Resolvably decomposing complete equipartite graphs minus a one-factor into cycles of uniform even length, Ars Combin. 110 (2013), 435-445.

[15] D. G. Hoffman and P. J. Schellenberg, The existence of $C_{k}$-factorizations of $K_{2 n}-F$, Discrete Math. 97 (1991), 243-250, doi:10.1016/0012-365x(91)90440-d.

[16] M. S. Keranen and S. Özkan, The Hamilton-Waterloo problem with 4-cycles and a single factor of $n$-cycles, Graphs Combin. 29 (2013), 1827-1837, doi:10.1007/s00373-012-1231-6.

[17] M. S. Keranen and A. Pastine, A generalization of the Hamilton-Waterloo problem on complete equipartite graphs, J. Combin. Des. 25 (2017), 431-468, doi:10.1002/jcd.21560.

[18] H. Lei and H. Shen, The Hamilton-Waterloo problem for Hamilton cycles and triangle-factors, J. Combin. Des. 20 (2012), 305-316, doi:10.1002/jcd.20311.

[19] J. Liu, A generalization of the Oberwolfach problem and $C_{t}$-factorizations of complete equipartite graphs, J. Combin. Des. 8 (2000), 42-49, doi:10.1002/(sici)1520-6610(2000)8:1<42:: aid-jcd6 $>3.0 . c 0 ; 2-r$.

[20] J. Liu, The equipartite Oberwolfach problem with uniform tables, J. Comb. Theory Ser. A 101 (2003), 20-34, doi:10.1016/s0097-3165(02)00011-0.

[21] U. Odabaşıand S. Özkan, The Hamilton-Waterloo problem with $C_{4}$ and $C_{m}$ factors, Discrete Math. 339 (2016), 263-269, doi:10.1016/j.disc.2015.08.013.

[22] D. K. Ray-Chaudhuri and R. M. Wilson, Solution of Kirkman's schoolgirl problem, in: T. S. Motzkin (ed.), Combinatorics, American Mathematical Society, Providence, Rhode Island, volume XIX of Proceedings of Symposia in Pure Mathematics, 1971 pp. 187-203, proceedings of the Symposium in Pure Mathematics of the American Mathematical Society held at the University of California, Los Angeles, California, March 21 - 22, 1968.

[23] G. Rinaldi and T. Traetta, Graph products and new solutions to Oberwolfach problems, Electron. J. Combin. 18 (2011), \#P52 (17 pages), https://www.combinatorics.org/ ojs/index.php/eljc/article/view/v18ilp52.

[24] T. Traetta, A complete solution to the two-table Oberwolfach problems, J. Comb. Theory Ser. A 120 (2013), 984-997, doi:10.1016/j.jcta.2013.01.003.

[25] L. Wang, F. Chen and H. Cao, The Hamilton-Waterloo problem for $C_{3}$-factors and $C_{n}$-factors, J. Combin. Des. 25 (2017), 385-418, doi:10.1002/jcd.21561. 